\title{
Accretions of various types of dark energies onto Morris-Thorne wormhole
}

\author{
Ujjal Debnath $^{\mathrm{a}}$ \\ Department of Mathematics, Bengal Engineering and Science University, Shibpur, Howrah 711 103, India
}

Received: 15 October 2013 / Accepted: 23 April 2014 / Published online: 6 May 2014

(c) The Author(s) 2014. This article is published with open access at Springerlink.com

\begin{abstract}
In this work, we have studied the accretion of dark energies onto a Morris-Thorne wormhole. Previously, in ref. (González-Díaz, arXiv:hep-th/0607137), it was shown that for quintessence like dark energy, the mass of the wormhole decreases, and for phantom like dark energy, the mass of the wormhole increases. We have assumed two types of dark energy: the variable modified Chaplygin gas and the generalized cosmic Chaplygin gas. We have found the expression of the wormhole mass in both cases. We have found the mass of the wormhole at late universe and this is finite. For our choices of the parameters and the function $B(a)$, these models generate only quintessence dark energy (not phantom) and so the wormhole mass decreases during the evolution of the universe. Next we have assumed the five kinds of parametrizations of well-known dark-energy models. These models generate both quintessence and phantom scenarios i.e., phantom crossing models. So if these dark energies accrete onto the wormhole, then for the quintessence stage, the wormhole mass decreases up to a certain value (a finite value) and then again increases to an infinite value for the phantom stage during whole evolution of the universe. That means that if the five kinds of DE accrete onto a wormhole, the mass of the wormhole decreases up to a certain finite value and then increases in the late stage of the evolution of the universe. We have also shown these results graphically.
\end{abstract}

\section{Introduction}

Due to recent observations it is strongly believed that the universe is experiencing an accelerated expansion. The type Ia supernovae and cosmic microwave background (CMB) [1,2] observations have shown evidence to support cosmic acceleration. This acceleration is caused by some unknown matter, which has the property of having positive energy density and negative pressure satisfying $\rho+3 p<0$, dubbed as "dark

\footnotetext{
a e-mail: ujjaldebnath@yahoo.com; ujjal@iucaa.ernet.in
}

energy' (DE) [3-6]. If $\rho+p<0$, it is dubbed 'phantom energy'. The combined astrophysical observations suggest that the universe is spatially flat and the dark energy occupies about $70 \%$ of the total energy of the universe; the contribution of dark matter is $\sim 26 \%$; the baryon contribution is $4 \%$, and we have negligible radiation. A cosmological property in which there is an infinite expansion in scale factor in a finite time is termed a 'big rip'. In phantom cosmology, the big rip is a kind of future singularity in which the energy density of phantom energy will become infinite in a finite time. To realize the big rip scenario the condition $\rho+p<0$ alone is not sufficient [7]. Distinct data on supernovas showed that the presence of phantom energy with $-1.2<w<-1$ in the universe is highly likely [8]. In this case the cosmological phantom energy density grows at large times and disrupts finally all bounded objects up to subnuclear scale.

A wormhole is a feature of space that is essentially a 'shortcut' from one point in the universe to another point in the universe, allowing travel between them that is faster than it would take light to make the journey through normal space. So the wormholes are tunnels in spacetime geometry that connect two or more regions of the same spacetime or two different spacetimes [9]. Wormholes may be classified into two categories-Euclidean wormholes and Lorentzian wormholes. The Euclidean wormholes arise in Euclidean quantum gravity; the Lorentzian wormholes [10] are static spherically symmetric solutions of Einstein's general relativistic field equations [11]. In order to support such exotic wormhole geometries, the matter should violate the energy conditions (null, weak and strong), but the average null energy condition is satisfied in wormhole geometries [12-14]. For small intervals of time, the weak energy condition (WEC) can be satisfied [15]. Also the traversable wormhole solutions of the field equations are obtained [16]. Recently evolving wormhole solutions and their implications have been discussed by several authors [17-24].

The equations of motion for steady-state spherical symmetric flow of matter into or out of a condensed object 
(e.g. neutron stars, 'black holes', etc.) are discussed by Michel [25] and also he obtained an analytic relativistic accretion solution onto the static Schwarzschild black hole. The accretion of phantom dark energy onto a Schwarzschild black hole was first modeled by Babichev et al. [26,27]. They established that the black hole mass will gradually decrease due to a strong negative pressure of the phantom energy and finally all the masses tend to zero near the big rip, where it will disappear. Accretion of a phantom like variable modified Chaplygin gas onto Schwarzschild black hole was studied by Jamil [28] who showed that the mass of the black hole will decrease when accreting fluid violates the dominant energy condition and otherwise will increase. Also the accretion of dark energy onto the more general KerrNewman black hole was studied by Madrid et al. [29] and Bhadra et al. [30]. Till now, several authors [31-45] have discussed the accretion of various components of dark energy onto black holes. Recently, there has been a great interest in the study of dark-energy accretion onto a static wormhole [46-48]. The phantom energy accretion onto a wormhole is discussed by González-Díaz [49]. Madrid et al. [50] studied the dark-energy accretion onto black holes and worm holes phenomena could lead to unexpected consequences, allowing even the avoidance of the considered singularities. Also Martín-Moruno et al. [51] have considered a general formalism for the accretion of dark energy onto astronomical objects, black holes, and wormholes. It has been shown that in models with four dimensions or more, any singularity with a divergence in the Hubble parameter may be avoided by a big trip, if it is assumed that there is no coupling between the bulk and this accreting object. If this is not the case in more than four dimensions, the evolution of the cosmological object depends on the particular model. The dark-energy accretion onto a wormhole in an accelerating universe has also been discussed [52,53] recently.

In the following section, we assume the Morris-Thorne static wormhole in the presence of a dark-energy filled universe. If dark energy accretes onto the wormhole, the rate of change of mass of the wormhole is expressed in terms of the density and pressure of the dark energy and we also find the expression of the wormhole mass in terms of the density. Our main motivation of the work is to examine the nature of the mass of the wormhole during the expansion of the universe if several kinds of dark energies accrete around the wormhole. The candidates of dark energy are assumed to be the variable modified Chaplygin gas (VMCG) and the generalized cosmic Chaplygin gas (GCCG). Also we have assumed some kinds of parametrizations of dark-energy candidates. The mass of the wormhole has been calculated for all types of dark energies and its nature has been analyzed during the expansion of the universe. Finally, we give some concluding remarks of the whole work.

\section{Accretion phenomena of dark energy onto wormhole}

Let us consider spherically symmetrical accretion of the dark energy onto the wormhole. We consider a non-static spherically symmetric Morris-Thorne wormhole metric [9] given by

$\mathrm{d} s^{2}=-e^{\Phi(r)} \mathrm{d} t^{2}+\frac{\mathrm{d} r^{2}}{1-\frac{K(r)}{r}}+r^{2}\left(\mathrm{~d} \theta^{2}+\sin ^{2} \theta \mathrm{d} \phi^{2}\right)$

where the functions $K(r)$ and $\Phi(r)$ are the shape function and redshift function, respectively, of the radial co-ordinate $r$. If $K\left(r_{0}\right)=r_{0}$, the radius $r_{0}$ is called the wormhole throat radius. So we want to consider the outward region such that $r_{0} \leq r<\infty$.

A proper dark-energy accretion model for wormholes should be obtained by generalizing the Michel theory [25] to the case of wormholes. Such a generalization has been already performed by Babichev et al. $[26,27]$ for the case of dark-energy accretion onto Schwarzschild black holes. We shall follow now the procedure used by Babichev et al. $[26,27]$, adapting it to the case of a Morris-Thorne wormhole [52]. For this purpose, we consider the energy-momentum tensor for the dark energy (DE) in the form of a perfect fluid having the $\operatorname{EoS} p=p(\rho)$, which is

$T_{\mu \nu}=(\rho+p) u_{\mu} u_{\nu}+p g_{\mu \nu}$

where $\rho$ and $p$ are the energy density and pressure of the dark energy, respectively, and $u^{\mu}=\frac{\mathrm{d} x^{\mu}}{\mathrm{d} s}$ is the fluid fourvelocity satisfying $u^{\mu} u_{\mu}=-1$. We assume that the in-falling dark energy fluid does not disturb the spherical symmetry of the wormhole. Now we assume $\Phi(r)=0$. The relativistic Bernoulli equation after the time component of the energymomentum conservation law $T_{; \nu}^{\mu \nu}=0$ provides the first integral of motion for static, spherically symmetric accretion onto a wormhole which yields [52]

$M^{-2} r^{2} u(\rho+p)\left(1-\frac{K(r)}{r}\right)^{-1}\left(u^{2}+\frac{K(r)}{r}-1\right)^{\frac{1}{2}}=C_{1}$

where $M$ is the exotic mass of the wormhole, $u=\frac{\mathrm{d} r}{\mathrm{~d} s}$ (> 0 ) is the radial component of the velocity four-vector and the integration constant $C_{1}$ has the dimension of the energy density.

Moreover, the second integration of motion is obtained from the projection of the conservation law for the energymomentum tensor onto the fluid four-velocity, $u_{\mu} T_{; \nu}^{\mu \nu}=0$, which gives [52]

$M^{-2} r^{2} u\left(1-\frac{K(r)}{r}\right)^{-\frac{1}{2}} \exp \left[\int_{\rho_{\infty}}^{\rho} \frac{\mathrm{d} \rho}{\rho+p(\rho)}\right]=C_{2}$ 
where $C_{2}(>0)$ is a dimensionless integration constant, $\rho_{\infty}$ is the dark-energy density at infinity. Further the value of the constant $C_{2}$ can be evaluated for different dark energy models. The critical point for this accretion process has been found in [54].

The rate of change of the mass $\dot{M}$ of the exotic wormhole is computed by integrating the flux of the dark energy over the entire two-dimensional surface of the wormhole, i.e., $\dot{M}=\oint T_{t}^{r} \mathrm{~d} S$, where $T_{t}^{r}$ represents the radial component of the energy-momentum densities and $\mathrm{d} S=\sqrt{-g} \mathrm{~d} \theta \mathrm{d} \phi=$ $r^{2} \sin \theta \mathrm{d} \theta \mathrm{d} \phi[26,55]$ is the element of the wormhole surface. Using the above equations we obtain the rate of change of mass as [52]

$\dot{M}=-4 \pi Q M^{2}\left(1-\frac{K(r)}{r}\right)^{\frac{1}{2}}(\rho+p)$

where $Q$ is a positive constant. For the relevant asymptotic regime $r \rightarrow \infty$, the above equation reduces to

$\dot{M}=-4 \pi Q M^{2}(\rho+p)$

We see that the rate for the wormhole exotic mass due to the accretion of dark energy becomes exactly the negative of the similar rate in the case of a Schwarzschild black hole, asymptotically. Since the Morris-Thorne wormhole is static, the mass of the wormhole depends on $r$ only. When some fluid accretes outside the wormhole, the mass function $M$ of the wormhole is considered as a dynamical mass function and hence it should be a function of time also. So $\dot{M}$ of Eq. (6) is time dependent and the increasing or decreasing of the wormhole mass $M$ sensitively depends on the nature of the fluid which accretes upon the wormhole. If $\rho+p<0$, i.e., for phantom dark-energy accretion, the mass of the wormhole increases but if $\rho+p>0$, i.e., for non-phantom dark-energy accretion, the mass of the wormhole decreases [52]. In the following, we shall assume different types of dark-energy models, such as variable modified Chaplygin gas, generalized cosmic Chaplygin gas and parametrizations of some kinds of well-known dark energy models. The nature of the mass function of the wormhole will be analyzed for present and future stages of expansion of the universe when the above types of dark energies are accreting upon the wormhole.

\subsection{Variable modified Chaplygin gas as dark-energy model}

We consider the background spacetime to be spatially flat, represented by the homogeneous and isotropic FRW model of the universe which is given by

$$
\mathrm{d} s^{2}=-\mathrm{d} t^{2}+a^{2}(t)\left[\mathrm{d} r^{2}+r^{2}\left(\mathrm{~d} \theta^{2}+\sin ^{2} \theta \mathrm{d} \phi^{2}\right)\right]
$$

where $a(t)$ is the scale factor. We assume that the universe is filled with a VMCG and the EoS is [56] given by

$p=A \rho-\frac{B(a)}{\rho^{\alpha}}$ with $0 \leq \alpha \leq 1, A$ is constant $>0$.

Here $B(a)$ is the function of scale factor $a$ and for simplicity we choose $B(a)=B_{0} a^{-n}$, where $B_{0}>0$ and $n>0$ are constants.

The Einstein equations for the FRW universe are (choosing $8 \pi G=c=1$ )

$H^{2}=\frac{1}{3} \rho$,

$\dot{H}=-\frac{1}{2}(p+\rho)$.

The conservation equation satisfied by the dark-energy model VMCG is

$\dot{\rho}+3 H(\rho+p)=0$

where $H=\frac{\dot{a}}{a}$ is the Hubble parameter. Using Eqs. (7) and (10), we have the solution of $\rho$ :

$\rho=\left[\frac{3(1+\alpha) B_{0}}{\{3(1+\alpha)(1+A)-n\}} \frac{1}{a^{n}}+\frac{C}{a^{3(1+A)(1+\alpha)}}\right]^{\frac{1}{1+\alpha}}$

where $C>0$ is an arbitrary integration constant and 3(1+ A) $(1+\alpha)>n$, for positivity of the first term.

Using Eqs. (6), (9), and (11), we have [51,57]

$\dot{M}=\frac{4 \pi}{\sqrt{3}} Q M^{2} \rho^{-\frac{1}{2}} \dot{\rho}$,

which integrates to yield

$M=\frac{M_{0}}{1-\frac{8 \pi Q M_{0}}{\sqrt{3}}\left(\sqrt{\rho}-\sqrt{\rho_{0}}\right)}$

where $M_{0}$ and $\rho_{0}$ are the present values of the wormhole mass and density of the dark energy, respectively. Using Eq. (9), we get the mass function in terms of the Hubble parameter $H$ in the form [51,57]

$M=\frac{M_{0}}{1-8 \pi Q M_{0}\left(H-H_{0}\right)}$

where $H_{0}$ is the present value of the Hubble parameter. In the late stage of the universe i.e., when $a$ is very large $(z \rightarrow-1)$, the mass of the wormhole will be

$M=\frac{M_{0}}{1+\frac{8 \pi Q M_{0}}{\sqrt{3}} \sqrt{\rho_{0}}}$.

If we put the solution $\rho$ from Eq. (12) in Eq. (14), the mass of the wormhole $M$ can be expressed in terms of the scale factor $a$ and then use the formula of the redshift $z=\frac{1}{a}-1$, 


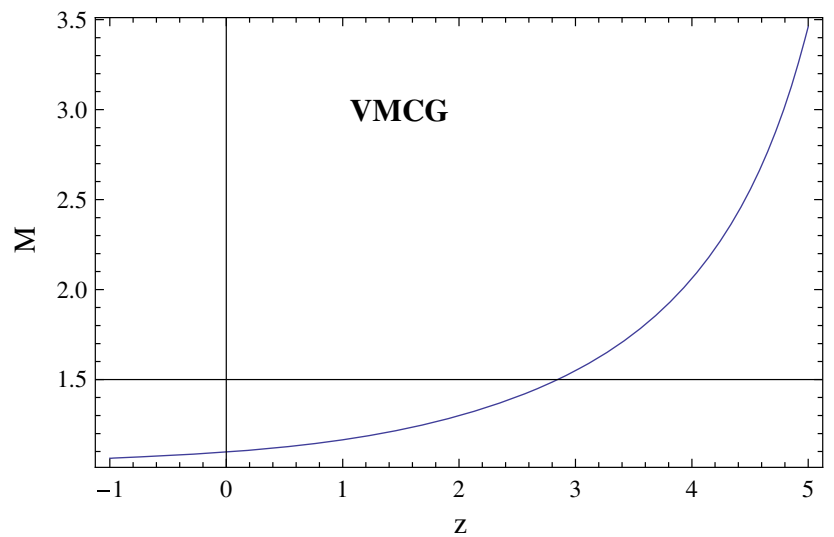

Fig. 1 The variations of the wormhole mass $M$ against the redshift $z$ for the VMCG models

$M$ will be defined in terms of the redshift $z . M$ vs. $z$ is drawn in Fig. 1. For our choice of the function $B(a)$, the VMCG gives only the quintessence dark energy, not the phantom dark energy. So the mass of the wormhole always decreases for our case. From the figure, we see that $M$ decreases with $z$ decreasing. So the mass of the wormhole decreases if the VMCG accretes onto the wormhole.

\subsection{Generalized cosmic Chaplygin gas as the dark-energy model}

A new version of the Chaplygin gas which is known as GCCG $[58,59]$ obeys the equation of state

$p=-\rho^{-\alpha}\left[C+\left(\rho^{1+\alpha}-C\right)^{-w}\right]$

where $C=\frac{A^{\prime}}{(1+w)}-1, A^{\prime}$ is either a positive or a negative constant, $-l<w<0,0 \leq \alpha \leq 1$, and $l>1$. The EOS reduces to that of the current Chaplygin unified models for dark matter and dark energy in the limit $w \rightarrow 0$ and satisfies the conditions: (i) it becomes a de Sitter fluid at late time and when $w=-1$, (ii) it reduces to $p=w \rho$ in the limit that the Chaplygin parameter $A^{\prime} \rightarrow 0$, (iii) it also reduces to the EOS of current Chaplygin unified dark matter models at high energy density, and (iv) the evolution of the density perturbations derived from the chosen EOS becomes free from the pathological behavior of the matter power spectrum for physically reasonable values of the involved parameters at late time. This EOS shows the dust era in the past and $\Lambda \mathrm{CDM}$ in the future.

From the conservation equation (10), we have the expression for the energy density of GCCG in the form $[58,59]$

$\rho=\left[C+\left(1+\frac{B}{a^{3(1+\alpha)(1+w)}}\right)^{\frac{1}{1+w}}\right]^{\frac{1}{1+\alpha}}$.

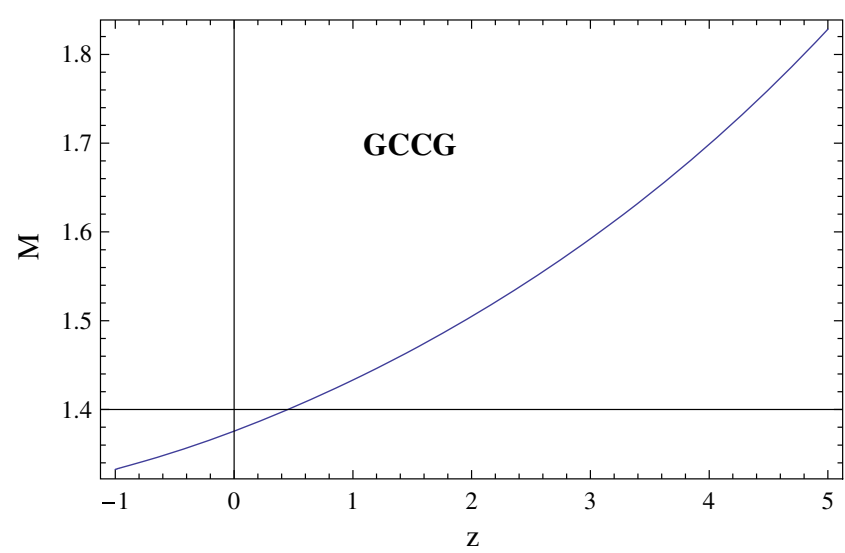

Fig. 2 The variations of the wormhole mass $M$ against the redshift $z$ for the GCCG models

In the late stage of the universe i.e., $a$ is very large $(z \rightarrow-1)$, and the mass of the wormhole will be

$M=\frac{M_{0}}{1-\frac{8 \pi Q M_{0}}{\sqrt{3}}\left((1+C)^{\frac{1}{2(1+\alpha)}}-\sqrt{\rho_{0}}\right)}$.

If we put the solution $\rho$ from Eq. (18) in Eq. (14), the mass of the wormhole $M$ can be expressed in terms of the scale factor $a$ and hence $M$ will be defined in terms of the redshift $z . M$ vs. $z$ is drawn in Fig. 2. The GCCG gives only the quintessence dark energy, not the phantom dark energy. So the mass of the wormhole always decreases for our case. From the figure, we see that $M$ decreases with $z$ decreasing. So the mass of the wormhole decreases if the GCCG accretes onto the wormhole.

\subsection{Some parameterizations of dark-energy models}

In an astrophysical sense, the dark energy is popular as it has a redshift parametrization (i.e., taking the redshift $z$ as the variable parameter of the EoS only) of the EoS as $p(z)=$ $w(z) \rho(z)$. The EoS parameter $w$ is currently constrained by distance measurements of type Ia supernovae and the current observational data constrain the range of EoS as $-1.38<$ $w<-0.82$ [60]. Recently, the combination of WMAP3 and Supernova Legacy Survey data shows a significant constraint on the EOS, $w=-0.97_{-0.09}^{+0.07}$ for the DE, in a flat universe [61]. Two mainstream families of red shift parametrizations are considered here, viz.,

(i) Family I: $w(z)=w_{0}+w_{1}\left(\frac{z}{1+z}\right)^{n}$. In this case, the conservation equation (11) gives the solution

$$
\rho=\rho_{0}(1+z)^{3\left(1+w_{0}\right)} e^{3(-1)^{-n} w_{1}\{\text { Beta }[1+z,-n, 1+n]+\pi \operatorname{cosec} n \pi\}} .
$$


(ii) Family II: $w(z)=w_{0}+w_{1} \frac{z}{(1+z)^{n}}$. The solution becomes

$$
\rho=\rho_{0}(1+z)^{3\left(1+w_{0}\right)} e^{-\frac{3 w_{1}}{n(n-1)}\left[\frac{1+n z}{(1+z)^{n}}-1\right]}
$$

Here $w_{0}$ and $w_{1}$ are two unknown parameters, which can be constrained by recent observations and $n$ is a natural number. For different values of $n$, we will get the following three models of well-known parametrizations (Models I, II, and III). We shall also assume two other parametrizations (Models IV and V). The following models generate both quintessence $(w(z)>-1)$ and phantom $(w(z)<-1)$ dark energies for some suitable choices of the parameters. So a phantom divide is possible at the $\Lambda \mathrm{CDM}$ stage $w(z)=-1$. At the first stage, there occurs quintessence and in the late stage there occurs phantom dark energy. So for the quintessence stage, the mass of the wormhole decreases and is decreasing up to a certain limit (of the mass) and then again at the phantom stage, the mass of the wormhole increases.

- Model I (Linear): For $n=0$, family II reduces to the parametrization form $w(z)=w_{0}+w_{1} z$ [62]. This is known as the 'Linear' parametrization. For the linear parametrization, the solution becomes

$$
\rho=\rho_{0}(1+z)^{3\left(1+w_{0}-w_{1}\right)} e^{3 w_{1} z} .
$$

The above model generates phantom energy if $w(z)<-1$ i.e., $z<-\frac{1+w_{0}}{w_{1}}$ provided $w_{1}>0$ and $w_{1}-w_{0}>1$. If we drop this restriction, this model gives the quintessencetype dark energy. Since this model is the phantom crossing model, if this dark energy accretes onto a wormhole, for the quintessence era, the wormhole mass decreases up to a certain limit and after that, for the phantom era, the mass of the wormhole increases. We have shown this scenario in Fig. 3. We see that the wormhole mass $M$ decreases with redshift $z$ decreasing up to certain stage of $z$ ( $\Lambda \mathrm{CDM}$

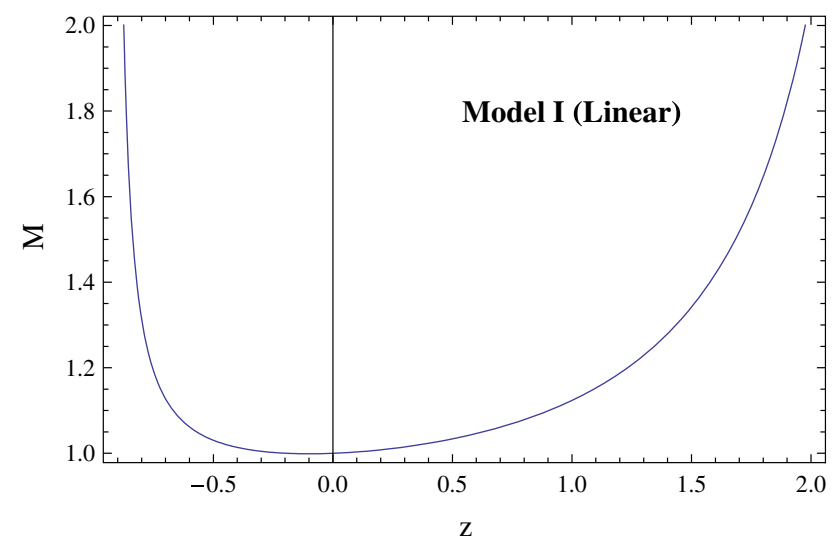

Fig. 3 The variations of the wormhole mass $M$ against the redshift $z$ for Models I, respectively

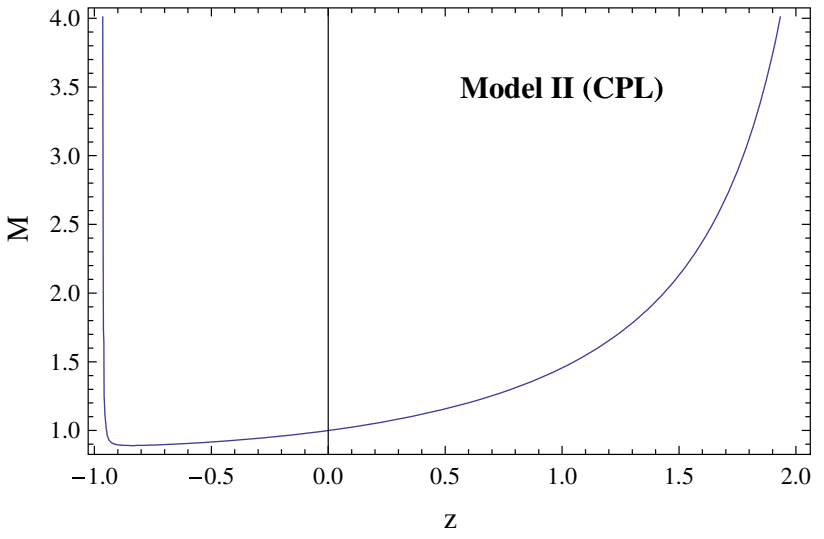

Fig. 4 The variations of the wormhole mass $M$ against the redshift $z$ for Models II, respectively

stage) and then $M$ increases (phantom era) as the universe expands.

- Model II (CPL): For $n=1$, both families I and II lead to the parametrization $w(z)=w_{0}+w_{1} \frac{z}{1+z}$. This is known as 'CPL' parametrization $[63,64]$. The solution becomes

$$
\rho=\rho_{0}(1+z)^{3\left(1+w_{0}+w_{1}\right)} e^{-\frac{3 w_{1} z}{1+z}} .
$$

The above model generates phantom energy if $w(z)<-1$, i.e., $z<-\frac{1+w_{0}}{1+w_{1}}$ provided $w_{1}>-1$ and $w_{1}-w_{0}>0$. If we drop this restriction, this model gives the quintessence type dark energy. This model is also the phantom crossing model. From Fig. 4, we see that the wormhole mass $M$ decreases for redshift $z$ decreasing up to certain stage of $z$ ( $\Lambda$ CDM stage) and then $M$ increases (phantom era) as the universe expands.

- Model III (JBP): For family $I I, n=2$ gives the parametrization $w(z)=w_{0}+w_{1} \frac{z}{(1+z)^{2}}$. This is known as 'JBP' parametrization [65]. The solution is

$$
\rho=\rho_{0}(1+z)^{3\left(1+w_{0}\right)} e^{\frac{3 w_{1} z^{2}}{2(1+z)^{2}}}
$$

The above model generates phantom energy if $w(z)<-1$, i.e., $z<-1+\frac{\sqrt{4\left(1+w_{0}\right) w_{1}+w_{1}^{2}}}{2\left(1+w_{0}\right)}$, provided $w_{0}>-1$ and $w_{1}<-4\left(1+w_{0}\right)$. If we drop this restriction, this model gives the quintessence type dark energy. This model is also the phantom crossing model. From Fig. 5, we see that the wormhole mass $M$ decreases for redshift $z$ decreasing up to certain stage of $z$ ( $\Lambda \mathrm{CDM}$ stage) and then $M$ increases (phantom era) as the universe expands.

- Model IV: Another type of parametrization can be considered: $w(z)=-1+\frac{A_{1}(1+z)+2 A_{2}(1+z)^{2}}{3\left[A_{0}+A_{1}(1+z)+A_{2}(1+z)^{2}\right]}$, where $A_{0}, A_{1}$ and $A_{2}$ are constants $[66,67]$. This ansatz is exactly the one of the cosmological constant $w=-1$ for $A_{1}=A_{2}=0$ and DE models with $w=-2 / 3$ for $A_{0}=A_{2}=0$ and 


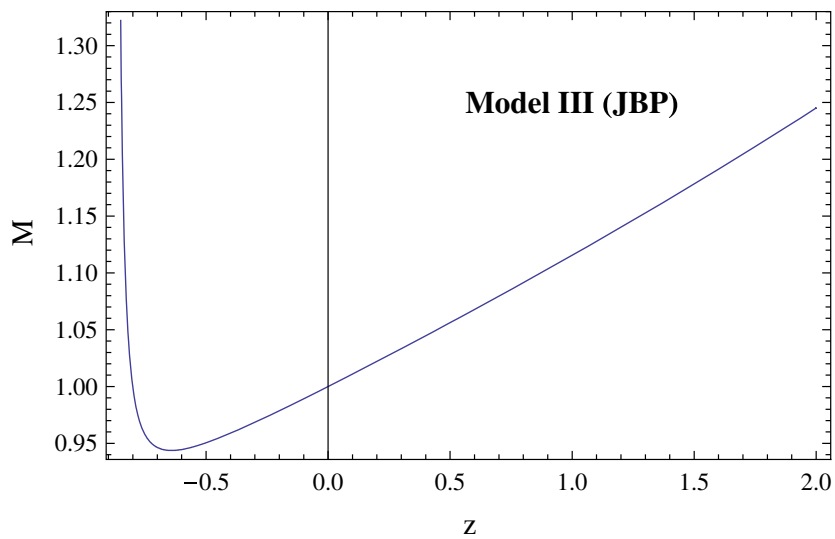

Fig. 5 The variations of the wormhole mass $M$ against the redshift $z$ for Models III, respectively

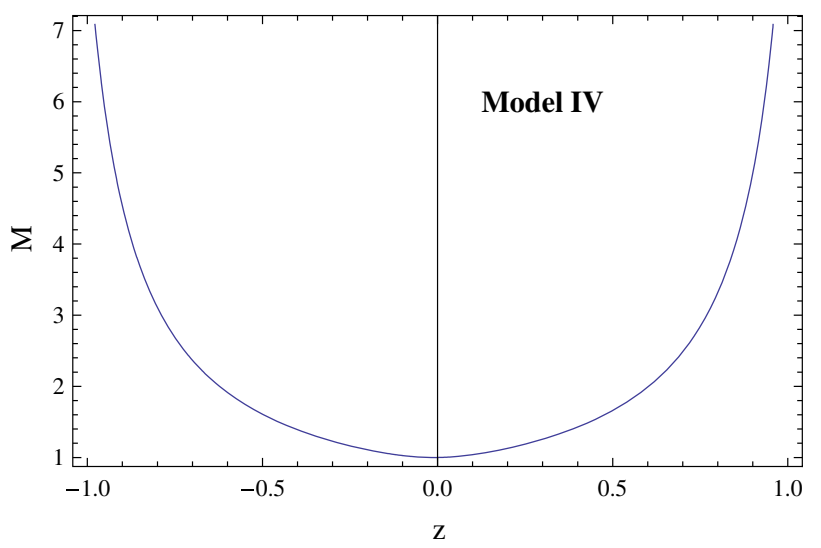

Fig. 6 The variations of the wormhole mass $M$ against the redshift $z$ for Models IV, respectively

$w=-1 / 3$ for $A_{0}=A_{1}=0$. In this case, we get the solution

$$
\rho=\frac{\rho_{0}\left[A_{0}+A_{1}(1+z)+A_{2}(1+z)^{2}\right]}{A_{0}+A_{1}+A_{2}} .
$$

The above model generates phantom energy if $w(z)<-1$, i.e., $z<-1-\frac{A_{1}}{A_{2}}$ provided $A_{0}<0, A_{1}>0, A_{2}<0$, and $A_{0}+A_{1}+A_{2}<0$. For this condition, $\rho$ is still positive. If we drop this restriction, this model gives the quintessence type dark energy. This model is also the phantom crossing model. From Fig. 6, we see that the wormhole mass $M$ decreases for redshift $z$ decreasing up to certain stage of $z$ and then $M$ increases (phantom era) as the universe expands.

- Model V: Another type of parametrization can be assumed: $w(z)=w_{0}+w_{1} \log (1+z)[68,69]$. The solution is obtained as

$$
\rho=\rho_{0}(1+z)^{3\left(1+w_{0}\right)} e^{\frac{3}{2} w_{1}[\log (1+z)]^{2}} .
$$

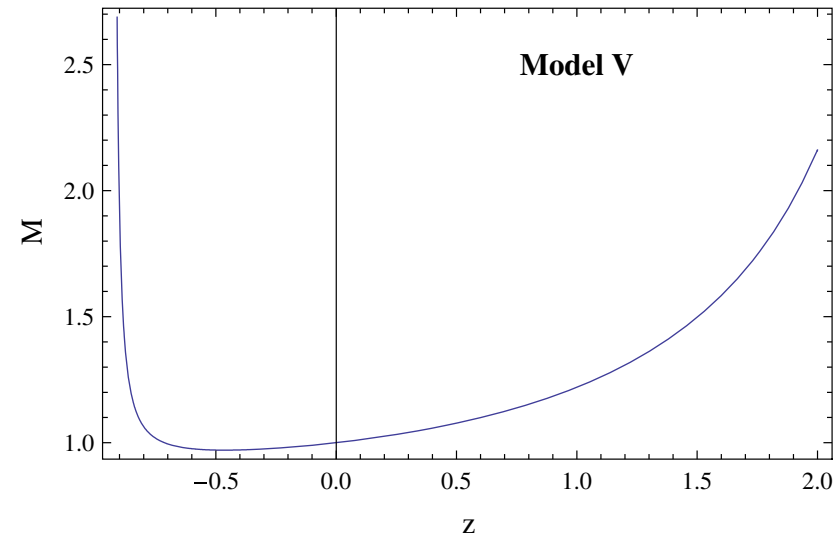

Fig. 7 The variations of the wormhole mass $M$ against the redshift $z$ for Models V, respectively

The above model generates phantom energy if $w(z)<-1$, i.e., $z<-1+e^{-\frac{w_{0}}{w_{1}}}$ provided $w_{1}>0$. If we drop this restriction, this model gives the quintessence type dark energy. This model is also the phantom crossing model. From Fig. 7, we see that the wormhole mass $M$ decreases for redshift $z$ decreasing up to certain stage of $z$ and then $M$ again increases (phantom era) as the universe expands.

\section{Discussions}

In this work, we have studied the accretion of the dark energies onto the Morris-Thorne wormhole. A proper darkenergy accretion model for wormholes has been obtained by generalizing the Michel theory [25] to the case of wormholes. Such a generalization has already been performed by Babichev et al. $[26,27]$ for the case of dark-energy accretion onto Schwarzschild black holes. We have followed the procedure used by Babichev et al. [26,27], adapting it to the case of a Morris-Thorne wormhole. Here we have assumed the redshift function $\Phi(r)=0$. Astrophysically, the mass of the wormhole is a dynamical quantity, so the nature of the mass function is important in our wormhole model for different dark-energy filled universe models. The sign of the time derivative of the wormhole mass depends on the signs of $\rho+p$. Previously Babichev et al. [26] have shown that the mass of black hole decreases due to phantom energy accretion. In ref. [52], it was shown that for quintessence like dark energy, the mass of the wormhole decreases; and for phantom like dark energy, the mass of the wormhole increases, which is the opposite of the behavior of the black hole mass.

We have assumed two recently proposed types of dark energy: the VMCG and the GCCG. We have found the expression of the wormhole mass in both cases. We have found the mass of the wormhole for the late universe and this is found to be finite. Our dark energy fluids violate the 
strong energy condition $(\rho+3 p<0$ in the late epoch), but do not violate the weak energy condition $(\rho+p>0)$. So the models drive only the quintessence scenario in the late epoch, but they do not generate the phantom epoch (in our choice). So the wormhole mass decreases during the evolution of the universe for these two dark-energy models. Since our considered dark-energy candidates do not violate the weak energy condition, the dynamical mass of the wormhole is decaying by the accretion of our considered dark energies, though the pressures of the dark energies are outside the wormhole. From Figs. 1 and 2, we observe that the wormhole mass decreases as $z$ increases for both VMCG and GCCG, which accrete onto the wormhole in our expanding universe. Next we have assumed five kinds of parametrizations (Models I-V) of well-known dark-energy models (some of them are linear, CPL, JBP models). These models generate both quintessence and phantom scenarios (phantom crossing models) for some restrictions of the parameters. So if these dark energies accrete onto the wormhole, then, for the quintessence stage, the wormhole mass decreases up to a certain value (a finite value) and then again increases to an infinite value for the phantom stage during the whole evolution of the universe. That means that if the five kinds of DE accrete onto a wormhole, the mass of the wormhole decreases up to a certain finite value and then increases in the late stage of the evolution of the universe. We also clearly shown these results graphically. Figures 3, 4, 5, 6, and 7 show that the mass of the wormhole first decreases to a finite value and then increases to an infinite value. In future work, it will be interesting to show the nature of the mass for various types of wormhole models if different kinds of dark energies accrete upon a wormhole in an accelerating universe also.

Acknowledgments The author is thankful to the Institute of Theoretical Physics, Chinese Academy of Science, Beijing, China, for providing the TWAS Associateship Programme under which part of the work was carried out. Also the author is thankful to CSIR, Govt. of India for providing research project grant (No. 03(1206)/12/EMR-II).

Open Access This article is distributed under the terms of the Creative Commons Attribution License which permits any use, distribution, and reproduction in any medium, provided the original author(s) and the source are credited.

Funded by $\mathrm{SCOAP}^{3}$ / License Version CC BY 4.0.

\section{References}

1. S.J. Perlmutter, et al., Nature 391, 51 (1998)

2. A.G. Riess, [Supernova Search Team Collaboration], et al., Astron. J. 116, 1009 (1998)

3. S. Briddle et al., Science 299, 1532 (2003)

4. D.N. Spergel et al., Astrophys. J. Suppl. 148, 175 (2003)

5. P.J.E. Peebles, B. Ratra, Astrophys. J. 325, L17 (1988)

6. R.R. Caldwell, R. Dave, P.J. Steinhardt, Phys. Rev. Lett. 80, 1582 (1998)

7. B. McInnes, J. High Energy Phys. 0208, 029 (2002)
8. U. Alam, V. Sahni, T.D. Saini, A.A. Starobinsky, Mon. Not. R. Astron. Soc. 354, 275 (2004)

9. M.S. Morris, K.S. Thorne, Am. J. Phys. 56, 395 (1988)

10. K. Thorne, Black Holes and Time Warps: Einstein's Outrageous Legacy (W. W. Norton \& Company, New York, 1995)

11. M. Visser, Lorentzian Wormholes: From Einstein to Hawking (AIP, New York 1995)

12. M. Visser, Phys. Rev. D 39, 3182 (1989)

13. M. Jamil, P.K.F. Kuhfittig, F. Rahaman, Sk. A Rakib, Eur. Phys. J. C 67, 513 (2010)

14. M. Jamil, M.U. Farooq, M.A. Rashid, Eur. Phys. J. C 59, 907 (2009)

15. S. Kar, Phys. Rev. D 49, 862 (1994)

16. O. Bertolami, R.Z. Ferreira, arXiv:1203.0523v1 [gr-qc]

17. S. Kar, D. Sahdev, Phys. Rev. D 53, 722 (1996)

18. L.A. Anchordoqui, D.F. Torres, M.L. Trobo, S.E.P. Bergliaffa, Phys. Rev. D 57, 829 (1998)

19. A.V.B. Arellano, F.S.N. Lobo, Class. Quantam Grav. 23, 5811 (2006)

20. M. Cataldo, S. del Campo, P. Minning, P. Salgado, Phys. Rev. D 79, 024005 (2009)

21. M. Cataldo, P. Labrana, S. del Campo, J. Crisostomo, P. Salgado, Phys. Rev. D 78, 104006 (2008)

22. M. Cataldo, P. Meza, P. Minning, Phys. Rev. D 83, 044050 (2011)

23. M. Farooq, M. Akbar, M. Jamil, AIP Conf. Proc. 1295, 176 (2010)

24. U. Debnath, M. Jamil, M. Akbar, arXiv:1202.1706v1 [physics.gen$\mathrm{ph}]$

25. F.C. Michel, Astrophys. Space Sci. 15, 153 (1972)

26. E. Babichev et al., Phys. Rev. Lett. 93, 021102 (2004)

27. E. Babichev, V. Dokuchaev, Y. Eroshenko, J. Exp. Theor. Phys. 100, 528-538 (2005)

28. M. Jamil, Eur. Phys. J. C62, 609 (2009)

29. J.A.J. Madrid, P.F. Gonźalez-Díaz, Grav. Cosmol. 14, 213 (2008)

30. J. Bhadra, U. Debnath, Eur. Phys. J. C 72, 1912 (2012)

31. S. Chakraborty, N. Mazumder, R. Biswas, Europhys. Lett. 91, 40007 (2010)

32. A.S. Majumdar, D. Gangopadhyay, L.P. Singh, arXiv:0709.3193v2 [gr-qc]

33. B. Nayaka, M. Jamil, arXiv:1107.2025v1 [gr-qc]

34. D. Dwivedee, B. Nayak, M. Jamil, L.P. Singh, arXiv:1110.6350v1 [gr-qc]

35. J.A.S. Lima, D.C. Guariento, J.E. Horvath, Phys. Lett. B 693, 218 (2010)

36. M. Sharif, G. Abbas, Chin. Phys. Lett. 28, 090402 (2011)

37. M. Sharif, G. Abbas, Chin. Phys. Lett. 29, 010401 (2012)

38. C.Y. Sun, Phys. Rev. D 78, 064060 (2008)

39. S.W. Kim, Y. Kang, Int. J. Mod. Phys. Conf. Ser. 12, 320 (2012)

40. P. Martín-Moruno et al, arXiv:0803.2005v1 [gr-qc]

41. M. Sharif, G. Abbas, arXiv:1106.2415v1 [gr-qc]

42. M.G. Rodrigues, A.E. Bernardiniz, arXiv:1208.1572v1 [gr-qc]

43. G. Abbas, arXiv:1303.6945v1 [gr-qc]

44. G. Abhas, arXiv:1309.0807v1 [gr-qc]

45. P. Martín-Moruno et al., arXiv:astro-ph/0603761

46. P.F. González-Díaz, Phys. Rev. Lett. 93, 071301 (2004)

47. V. Faraoni, W. Israel, Phys. Rev. D 71, 064017 (2005)

48. P.F. González-Díaz, arXiv:astro-ph/0404045

49. P.F. González-Díaz, Phys. Lett. B 632, 159 (2006)

50. J.A.J. Madrid, P. Martín-Moruno, arXiv:1004.1428 [astro-ph.CO]

51. P. Martín-Moruno, Phys. Lett. B 659, 40 (2008)

52. P.F. González-Díaz, arXiv:hep-th/0607137

53. P.F. González-Díaz and P. Martín-Moruno, arXiv:0704.1731v1 [astro-ph]

54. V.I. Dokuchaev, Y.N. Eroshenko, arXiv:1308.0896 [gr-qc]

55. L.D. Landau, E.M. Lifshitz, The Classical Theory of Fields (Pergamon, Oxford, 1975)

56. U. Debnath, Astrophys. Space Sci. 312, 295 (2007)

57. J.A.J. Madrid, Phys. Lett. B 634, 106 (2006) 
58. P.F. González-Díaz, Phys. Rev. D 68, 021303(R) (2003)

59. W. Chakraborty, U. Debnath, S. Chakraborty, Gravit. Cosmol. 13, 293 (2007)

60. A. Melchiorri, L. Mersini, M. Trodden, Phys. Rev. D 68, 043509 (2003)

61. Seljak, U., Slosar, A., McDonald, P., JCAP 0610014 (2006)

62. A.R. Cooray, D. Huterer, J. Astrophys. 513, L95 (1999)

63. M. Chevallier, D. Polarski, Int. J. Mod. Phys. D 10, 213 (2001)
64. Linder, E.V., Phys. Rev. Lett. 90, 091301(2003)

65. H.K. Jassal, J.S. Bagla, T. Padmanabhan, MNRAS 356, L11 (2005)

66. U. Alam, V. Sahni, T.D. Saini, A.A. Starobinski, Mon. Not. R. Astron. Soc. 354, 275 (2004)

67. U. Alam, V. Sahni, A.A. Starobinski, JCAP 0406, 008 (2004)

68. G. Efstathiou, Mon. Not. R. Astron. Soc. 310, 842 (1999)

69. R. Silva, J.S. Alcaniz, J.A.S. Lima, Int. J. Mod. Phys. D 16, 469 (2007) 